\title{
Military Blast Exposure and Chronic Neurodegeneration: Summary of Working Groups and Expert Panel Findings and Recommendations
}

\author{
${ }^{\star}$ Kelley A. Brix, David L. Brody, Jamie B. Grimes, ${ }^{3,4}$ Avraham Yitzhak, and Working Group Members **
}

\begin{abstract}
The potential relationship between chronic traumatic encephalopathy (CTE) and head injuries such as blast-related traumatic brain injury (TBI) is an important area of study, particularly for military and contact sports populations, yet little is known about this relationship. To address this topic, the Department of Defense (DoD) Blast Injury Research Program Coordinating Office organized the 2015 International State-of-the-Science Meeting, which brought together subject matter experts from the DoD, other federal agencies, academia, industry, foreign allies, and the sports community. Over the course of the meeting, this community of experts reached a consensus regarding the current body of knowledge and the future of the field. The overarching finding was that there is insufficient existing scientific evidence to link blast-related
\end{abstract}

${ }^{1}$ Defense Health Agency, Falls Church, Virginia

${ }^{2}$ Washington University School of Medicine, St. Louis, Missouri.

${ }^{3}$ Uniformed Services University of the Health Sciences, Bethesda, Maryland.

${ }^{4}$ Walter Reed National Military Medical Center, Bethesda, Maryland.

${ }^{5}$ Israeli Defense Force, Israel.

*Authors are listed alphabetically.

**Denes Agoston, ${ }^{4}$ Matt Aldag, ${ }^{8}$ Regina Armstrong, ${ }^{4}$ Peethambaran Arun, ${ }^{9}$ Michel Audette ${ }^{10}$ Debra Babcock, ${ }^{11}$ Carey Balaban, ${ }^{12}$ Rohan Banton, ${ }^{13}$ Patrick Bellgowan, ${ }^{11}$ David Borkholder, ${ }^{14}$ Steven Broglio, ${ }^{15}$ Elizabeth Brokaw, ${ }^{16}$ Robert Cantu, ${ }^{17}$ Walter Carr, ${ }^{18}$ Sandi Chapman, ${ }^{19}$ Joan Cmarik, ${ }^{18}$ Brian Colder, ${ }^{16}$ Jeff Colombe, ${ }^{16}$ David Cook, ${ }^{20}$ Tara Cozzarelli, ${ }^{21}$ Uade Olaghere da Silva,${ }^{22}$ Nitin Daphalapurkar,${ }^{23}$ Bernard Dardzinski ${ }^{4}{ }^{4}$ Tom DeGraba, ${ }^{24}$, Dr. James DeMar, ${ }^{9}$, Douglas DeWitt, ${ }^{25}$, Dara Dickstein, ${ }^{26}$, Josh Duckworth, ${ }^{4}$ Gregory Elder ${ }^{27}$ Reza Fazel-Rezai ${ }^{28}$ Michael Fine ${ }^{16}$ Gary Fiskum $^{29}$ Adam $^{33}$ Fournier $^{30}$, Shaleish Ganpule, ${ }^{23}$ Jessica Gill, ${ }^{31}$ John F. Glenn, ${ }^{18}$ Colin Greene, ${ }^{32}$ Nigel Greig, ${ }^{33}$ Chad Haering, ${ }^{34}$ Jason Harrington, ${ }^{35}$ Amy Hein, ${ }^{36}$ Kathy Helmick,${ }^{37}$ Ramona Hicks, ${ }^{72}$ Sidney Hinds, ${ }^{37}$ Stuart Hoffman, ${ }^{38}$ Dr. Iren Horkayne-Szakaly, ${ }^{2}$ Diego Iacono, ${ }^{4}$ Erick Ishii, ${ }^{38}$ Robert V. Jones, ${ }^{2}$ Ghodrat Karami, ${ }^{28}$ Daniel Krawczyk, ${ }^{19}$ Robert Labutta ${ }^{39}$ Robert Latta, ${ }^{40}$ Theresa Lattimore, ${ }^{2}$ Michael Leggieri, ${ }^{41}$ Fabio Leonessa, ${ }^{4}$ Alexander Lin, ${ }^{42}$ Geoffrey Ling, ${ }^{43}$ Matt Long, ${ }^{44}$ Kun Ping Lu, ${ }^{45}$ Stephanie Maxfield Panker, ${ }^{46}$ Joseph McCabe, ${ }^{4}$ Andrew Merkle, ${ }^{47}$ Philip Montenigro, ${ }^{17}$ Gary P. Mueller, ${ }^{4}$ Laurel Ng ${ }^{48}$ Savita Nigam, ${ }^{18}$ John O’Donnell, ${ }^{49}$ David Okonkwo, ${ }^{12}$ Ingrid Pauli, ${ }^{50}$ Daniel Perl, ${ }^{4}$ Elaine Peskind ${ }^{20}{ }_{53}$ Bryan Pfister, ${ }^{44}$ Mat Philippens,${ }^{51}$ Thuvan Piehler, ${ }^{13}$ Julie Proctor ${ }^{52}$ Andrzej Przekwas, ${ }^{53}$ Felicia Qashu, ${ }^{37}$ Murray Raskind, ${ }^{20}$ Alexander Razumovsky,${ }^{54}$ Jaques Reifman, ${ }^{18}$ Patricio Reyes, ${ }^{55}$ Paul Rigby, ${ }_{48}$ Marten Risling, ${ }^{56}$ Meghan Robinson, ${ }^{57}$ Tyler Rooks, ${ }^{58}$ Charles Rosen,${ }^{59}$ Gail Rosseau ${ }^{60}$ Wendy Sammons-Jackson, ${ }^{18}$ Anthony Santago, ${ }^{16}$ Richard Shoge,${ }^{18,61}$ Chandler Sours,${ }^{62}$ James Stone, ${ }^{63}$ Mike Templin, ${ }^{64}$ Victoria Tepe ${ }^{65}$ Peter Thielen, ${ }^{47}$ Mike Thomas, ${ }^{37}$ Thomas Timmes,${ }^{66}$ Frank Tortella, ${ }^{9}$ Laura Tucker, ${ }^{67}$ David Tweedie, ${ }^{33}$ Dale Vander Hamm, ${ }^{18}$ B. Christie Vu, ${ }^{18,36}$ Ying Wang,,${ }^{9}$ Therese West, ${ }^{37}$ Elisabeth Wilde, ${ }^{68}$ Adam Willis, ${ }^{69}$ John Wu, ${ }^{4}$ Laila Zai, ${ }^{70}$ Nikki Zander, ${ }^{13}$ James Zheng, ${ }^{71}$ and Mariusz Ziejewski. ${ }^{28}$

Working Group Members affiliations: ${ }^{8}$ Booz Allen Hamilton; ${ }^{9}$ Walter Reed Army Research Institute; ${ }^{10}$ Old Dominion University; ${ }^{11}$ National Institute of Neurological Disorders and Stroke, National Institutes of Health; ${ }^{12}$ University of Pittsburgh; ${ }^{13}$ US Army Research Laboratory; ${ }^{14}$ BlackBox Biometrics, Inc.; ${ }^{15}$ The University of Michigan; ${ }^{16}$ The MITRE Corporation; ${ }^{17}$ Boston University; ${ }^{18}$ US Army Medical Research and Materiel Command; ${ }^{19} \mathrm{Center}$ for Brain Health, The University of Texas at Dallas; ${ }^{20}$ VA Puget Sound Health Care System; ${ }^{21}$ Rehabilitation \& Regeneration Division, US Army Office of the Surgeon General; ${ }^{22}$ Naval Health Research Center; ${ }^{23}$ The Johns Hopkins University; ${ }^{24}$ National Intrepid Center of Excellence; ${ }^{25}$ University of Texas Medical Branch; ${ }^{26}$ Icahn School of Medicine at Mount Sinai; ${ }^{27}$ James J. Peters VA Medical Center; ${ }^{28}$ North Dakota State University; ${ }^{29}$ University of Maryland School of Medicine; ${ }^{30} \mathrm{PM}$ Soldier Protective Equipment; ${ }^{31}$ National Institutes of Health; ${ }^{32}$ US Army Joint Trauma Analysis and Prevention of Injuries in Combat; ${ }^{33}$ National Institute on Aging, National Institutes of Health; ${ }^{34}$ Natick Soldier Systems Center; ${ }^{35}$ U.S. Army Medical Materiel Agency; ${ }^{36}$ Congressionally Directed Medical Research Programs; ${ }^{37}$ Defense and Veterans Brain Injury Center; ${ }^{38}$ US Department of Veterans Affairs; ${ }^{39}$ US Army Medical and Materiel Development Activity; ${ }^{40} 711$ th Human Performance Wing, United States Air Force; ${ }^{41}$ DoD Blast Injury Research Program Coordinating Office; ${ }^{42}$ Brigham and Women's Hospital, Harvard Medical School; ${ }^{43}$ Defense Advanced Research Projects Agency; ${ }^{44}$ New Jersey Institute of Technology; ${ }^{45}$ Beth Israel Deaconess Medical Center, Harvard Medical School; ${ }^{46}$ Office of the Surgeon General; ${ }^{47}$ Johns Hopkins Applied Physics Lab; ${ }^{48}$ L-3 Applied Technologies, Inc; ${ }^{49}$ Marine Corps Systems Command; ${ }_{54}^{50}$ Navy Bureau of Medicine and Surgery; ${ }^{51}$ TNO Defence; ${ }_{55}^{52}$ University of Maryland, School of Medicine; ${ }^{53}$ CFD Research Corporation; ${ }^{54}$ Sentient Medical Systems; ${ }^{55}$ Phoenix VA Health Care System; ${ }^{56}$ Department of Neuroscience Karolinska Institutet; ${ }^{57}$ VA Boston Healthcare System; ${ }^{58}$ US Army Aeromedical Research Lab; ${ }^{59}$ Department of Neurosurgery, West Virginia University; ${ }^{60}$ Department of Neurosciences, Inova Fairfax Hospital; ${ }^{61}$ Joint Program Committee 5; ${ }^{62}$ Center for the Sustainment of Trauma and Readiness Skills; ${ }^{63}$ University of Virginia; ${ }^{64}$ SNBL USA; ${ }^{65}$ DoD Hearing Center of Excellence; ${ }^{66}$ US Army Center for Environmental Health Research; ${ }^{67}$ Center for Neuroscience and Regenerative Medicine; ${ }^{68}$ Michael E. DeBakey VA Medical Center, Baylor College of Medicine; ${ }^{69} 959$ CSPS/Brooke Army Medical Center; ${ }^{70}$ Applied Research Associates; ${ }^{71}$ PEO Soldier; ${ }^{72}$ One Mind. 
TBI with CTE. The meeting's Expert Panel also agreed on 13 additional findings describing research and knowledge gaps, clinical gaps, and research opportunities that, if addressed with focused effort, would further the understanding of the relationship between blast-related TBI and CTE. To this end, the Expert Panel also developed six recommendations for advancing research, each with short- and long-term goals. Among the six recommendations, the Expert Panel identified the first four as highest priority for addressing pressing research needs. These four high-priority recommendations include, in order of priority: (1) more collection and study of clinical neuropathology samples, (2) standardization of clinical diagnostic criteria, (3) development of clinically appropriate and standardized animal models, and (4) development of noninvasive serial assessment strategies (i.e., imaging or biospecimen biomarkers).

Keywords: blast-related injury; chronic traumatic encephalopathy (CTE); traumatic brain injury (TBI)

\section{Introduction}

$\mathbf{M}$ ORE Than 300,000 United States (US) service members have received a diagnosis of traumatic brain injury (TBI) since 2000. Most of these injuries are noncombat related (e.g., motor vehicle accidents, falls, training, sports), and the vast majority are mild. Blast-related, mild TBI (mTBI) is the most common injury that service members encounter in the combat settings of Iraq and Afghanistan, which leads to the following question: What are the long-term impacts of blast exposures, especially if there are multiple exposures that are not associated with acute symptoms? The question of a possible association between head impacts and chronic traumatic encephalopathy (CTE) in the military has a wellpublicized parallel to the contact sports community who, together with the Department of Defense (DoD), has made considerable investments in establishing multi-institutional and multi-disciplinary collaborative research programs focused on CTE.

CTE is described as a progressive neurodegenerative disorder affecting persons exposed to head injury and resulting in cognitive, behavioral, and/or motor deficits. Broad consensus on the existence of, and diagnostic criteria for, CTE, is the subject of active research. ${ }^{1-5}$ Still, multiple academic research groups and government organizations are gathering and analyzing evidence that may provide significant insights into the potential links between exposure to head injury and the development of CTE. ${ }^{6-11}$ Considering the number of athletes, service members, and veterans who are exposed to single and/or multiple brain injuries and/or subconcussive head impacts, CTE potentially represents a major public health issue.

To explore further the potential relationship between blastrelated trauma and CTE, The DoD Blast Injury Research Program Coordinating Office (PCO) hosted the 2015 International State-ofthe-Science (SoS) Meeting in November 2015. For additional information on the meeting, please see the introductory article in this supplement).

\section{Format and Process}

The SoS meeting series has been a forum for knowledge sharing, collaboration, and communication across blast injury research and stakeholder communities since 2009. Each PCO-hosted SoS meeting brings together a multi-disciplinary group of subject matter experts from across the DoD, other federal agencies, academia, industry, and allied nations to share their expertise and cutting-edge research on specific blast injury topics. These meetings leverage the expertise of the world's best scientists, engineers, and clinicians to identify blast injury knowledge gaps and recommend actions that will close the gaps.
The PCO's process for planning, hosting, and disseminating knowledge from the SoS meeting is shown in Figure 1. Briefly, to identify the topic for the 2015 meeting, the PCO requested input from DoD stakeholders who recognized CTE as a major civilian and military public health concern. Once CTE was selected as the topic of the meeting, a planning committee was established who worked to narrow the focus of the meeting, guide the specific research questions addressed within the literature review (see "The Biological Basis of Chronic Traumatic Encephalopathy following Blast Injury: A Literature Review" in this supplement), and identify expert panel members with the needed expertise.

During the meeting, speakers from government and academia summarized research community perspectives and research findings, which set the stage for the working group discussion and presentations that followed. These presentations sought to establish a shared understanding of the current scientific knowledge of blast-related trauma and the development of chronic neurodegeneration. Topic presentations discussed service member needs, the prevalence of military and civilian TBI, policy considerations, government program efforts, and perspectives from the research community. After the topic presentations, scientific presentations sharing research data, findings, and future recommendations served to inform the state of the science.

On the second and third days of the meeting, participants divided into six working groups, with each group chaired by an expert panel member: Col. Jamie Grimes, Drs. Stephen Ahlers, Kelley Brix, David Brody, William Stewart, and Lt. Col. Avraham Yitzak. The role of the expert panel members was to chair the working groups and encourage participation. Each working group discussed and answered the same set of five specific questions based on their expertise, findings from the literature review, and information from the topic and research presentations. After the working group discussions, participants reconvened and expert panel members presented the conclusions from their working groups.

After the 2015 SoS meeting, the expert panel convened during a closed session to identify key findings and recommendations that encapsulated the major discussion points and key ideas that recurred throughout the literature review, keynote, topic and scientific presentations, the poster sessions, and the focused working group sessions.

\section{Working Group Discussion Summary}

\section{Question \#1: What are the definitive pathological characteristics of neurodegeneration from repeated blast-induced trauma?}

What specific features could be used to characterize CTE as a unique neurodegenerative condition related to blast exposure? The working groups agreed that no definitive neuropathological characteristics specific to blast-induced neurodegeneration 


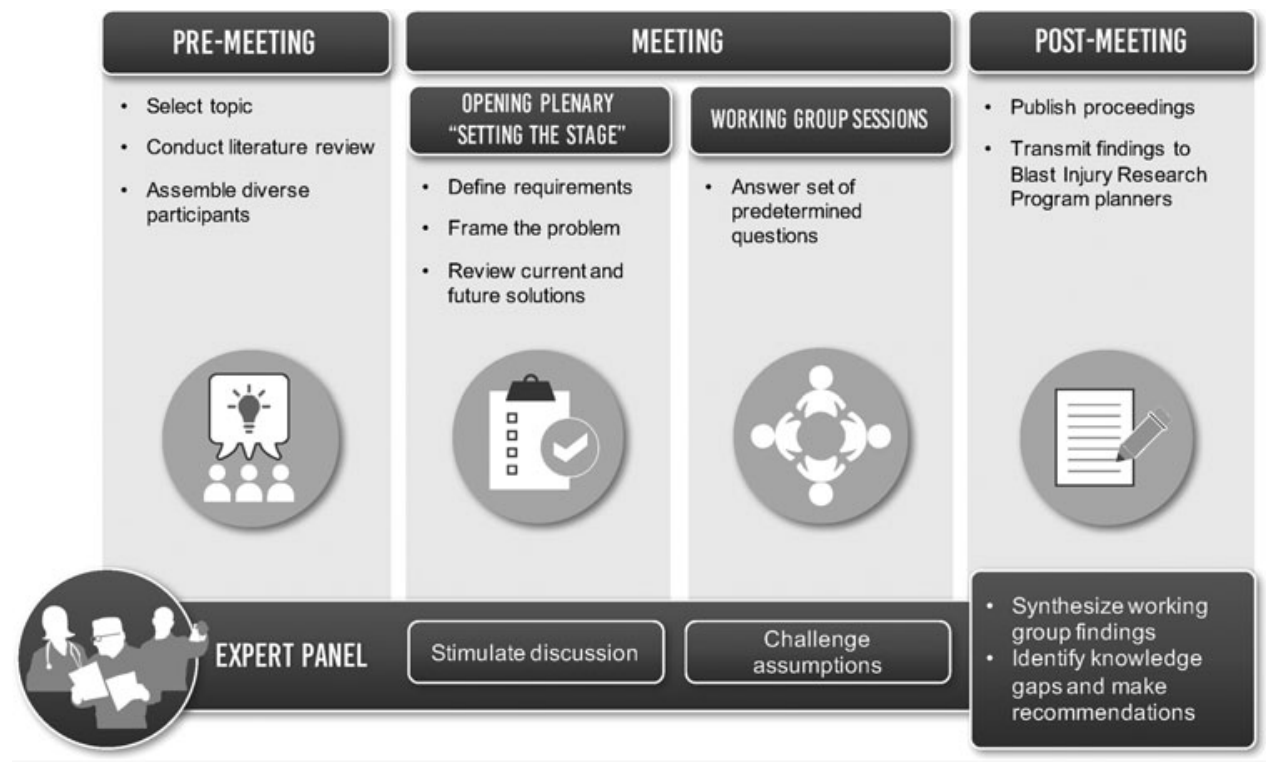

FIG. 1. International State-of-the-Science Meeting process for planning, hosting, and disseminating knowledge.

have been identified, which is largely attributed to a lack of blastinduced trauma cases available for study. The working groups supported existing National Institute of Neurological Disorders and Stroke (NINDS) consensus statements identifying diagnostic criteria for CTE (e.g., perivascular accumulation of phosphorylated tau in deep cortical sulci). This work was acknowledged as preliminary, however, given that the neuropathology described by consensus criteria had weak, if any, correlation with clinical phenotypes. In addition, the cohort examined to create this consensus criteria consisted of professional football players, not persons exposed to blast-induced trauma.

The working groups acknowledged that CTE may be part of a neurodegenerative spectrum of disease. Tau deposition was considered the feature most likely capable of uniquely characterizing CTE and associated neurodegeneration. Additional neuropathological analysis of brain tissues exposed to blunt (e.g., boxing, football) and blast injury is needed to determine whether the pathology of neurodegeneration after blast TBI is in the spectrum of CTE. Exploration of other neuropathological characteristics potentially associated with blast-related neurodegeneration and CTE is needed, including neuroinflammation, astrogliosis, microgliosis, axonal pathologies, amyloid deposition, cerebrovascular changes, alterations in deep sulci, and changes in cortical density and thickness.

One challenge to definitively identifying a neuropathological characteristic for diagnosing blast-associated neurodegeneration and CTE is the lack of human brain tissue available for analysis. To move the field forward, it is critical to encourage brain donation for existing and future clinical trials; donated tissue should also have well-characterized clinical, symptom, and exposure history with appropriate age-matched controls. Brain samples should be shared across laboratories for neuropathological analyses. When a solid understanding of the pathology of neurodegeneration from TBI is reached, then the field can move forward to create appropriate animal models of TBI that recapitulate what is observed in humans.

\section{Question \#2: What risk factors, both traumatic and nontraumatic, are predictive of CTE?}

What types of traumatic risk factors contribute to the development of CTE and at what frequency? How do factors such as overall resiliency, stress, age, genetics, and history of mental illness contribute to CTE development? How do blast exposure (e.g., frequency, severity) and other head trauma (e.g., impact, acceleration/deceleration, linear/rotational acceleration) correlate with progressive neurodegeneration?

Exposure to TBI can be considered a known risk factor for CTE; severity of trauma, frequency of trauma, and the time interval between multiple trauma exposures potentially are relevant characteristics of this risk factor. While clinically evident injuries are recognized to be a potential risk for neurodegeneration and CTE, it is possible that frequent subconcussive injuries also contribute to CTE. More frequent exposure to mTBI and shorter intervals between injuries are suggested to be associated with greater risk of neurodegeneration. Additional research is needed, however, to investigate the role of multiple (high frequency), subconcussive (low severity) exposures and the risk of CTE developing. Currently there is insufficient evidence to definitively identify risk factors for CTE other than TBI.

The working groups identified recommendations for future research on CTE risk factors, including (1) a better understanding of the physics of blast exposure; (2) improved linking of blast sensor data with blast physics; (3) prospective studies that adequately capture exposure data, lifetime medical and injury histories, and biological samples; and (4) animal models with physiologically relevant scaling of blast exposure characteristics and longer-term follow-up for measurement of outcomes.

The working groups highlighted potential traumatic and nontraumatic risk factors predictive of CTE. In addition to TBI exposure, traumatic risk factors include physical properties of blast, such as intensity or orientation. Potential nontraumatic contributing risk factors for CTE include (1) genetic predisposition; (2) periinjury factors (e.g., nutrition, hydration); (3) comorbid psychological or health factors (e.g., stress, sleep deprivation, substance abuse); (4) age at time of injury (first exposure at a younger age is more problematic); (5) comorbid psychological disorders; and (6) environmental factors.

Studies demonstrating a greater susceptibility to concussions in female athletes compared with male athletes and observations that service members generally take longer than athletes to recover from 
brain injury suggest further investigation is needed into the roles of gender and occupation as risk factors for CTE and neurodegeneration. Risk factors may contribute to neurodegeneration pathological mechanisms, including (1) acceleration of normal aging processes; (2) induction of neuronal death; (3) diffuse axonal injury combined with tau and cerebrovascular pathology; (4) initiation of inflammatory responses; (5) hypoxia/ischemia; and (6) neuroendocrine disturbances.

\section{Question \#3: What research is needed to explore the putative spatiotemporal development of CTE resulting from repeated blast exposure?}

What is the mechanism of injury in repeated exposure? Can dose-response curves be developed to establish a link between the dose of blast and brain injury, including frequency and severity of injury and rate of neurodegeneration? What retrospective and prospective human studies are required and what are the limitations of such studies?

The working groups agreed that large, prospective longitudinal clinical studies of military service members and/or veterans with, or at risk of, blast injury are needed to characterize the association between repeated blast exposure and the development of CTE. Cohorts of contact sport athletes could illuminate differences between impact- and blast-related exposures. Studies targeting specific military populations with well-controlled or well-known exposure environments could also be useful for assessment of longterm exposure risk. Comparison between combat blast-exposed military personnel and training-exposed control groups without combat experience could potentially identify the contribution of psychological factors to combat-related injury and CTE.

In addition to a commitment from funding agencies that is commensurate with the resources and time necessary for prospective studies, several policy-level actions for facilitating CTErelated research were identified. First, clinical data generated by Department of Veterans Affairs, likely the largest source of subjects who have been exposed to repeated blast injuries, should be made available for collaborative analysis. Second, because of a critical need for clinical neuropathological data, physician and military communities should encourage and enable brain donation and biospecimen collection in longitudinal studies that include subjects with TBI, neurodegenerative disorders, and mental health disorders. Third, data sharing of brain bank and biospecimen repositories should be strongly encouraged.

The working groups also agreed that the development of animal models is needed to better understand blast injury mechanisms and characterize dose-response curves. Pre-clinical approaches should emphasize clinical and operational relevance and seek to replicate human pathology to the greatest extent possible. Animal model studies should be conducted over longer time points compared with current experimental approaches to observe changes associated with chronic neurodegeneration. Given that the vast majority of pre-clinical work is performed in rodents, models using larger species with gyrencephalic brains should be adopted; existing findings observed in rodents also need to be validated in these larger models.

The working groups agreed that mechanisms underlying repeated exposure to blast injury are largely unknown; multiple biological mechanisms (e.g., neurotransmitter release, vascular injury, blood-brain barrier disruption, endoplasmic reticulum stress, white matter deformation, seizure activity), which may be associated with a particular blast feature (e.g., primary through quinary, force, duration, direction, frequency, reflection), are potential drivers of the neurodegenerative processes.

Research into the relationship between physical load and the mechanisms of biological response is needed. Existing physical modeling efforts are often oversimplified, rely on untested assumptions, and do not reflect complex biological processes fully (e.g., effects across solid-fluid interfaces of the brain). Current research gaps relevant to mechanism of injury include (1) determining whether tau and/or amyloid beta accumulation drive disease progression or whether they represent biological responses to injury; (2) understanding the pathophysiology priming responses to initial injury that result in increased vulnerability to subsequent injuries; and (3) determining mechanistic differences between multiple subconcussive events and a single traumatic event.

Development of dose-response curves is possible, but only over a longer-term timeframe. The potential for new clinical doseresponse studies is challenged by a lack of definitive dose measurement in humans, limited methods for quantitative assessment of neurodegeneration, as well as the reduction of deploymentrelated exposure among service members in recent years following Operation Enduring Freedom/Operation Iraqi Freedom. Animal models represent a substantive opportunity to characterize dose-response relationships and interrogate potential dosage variables, including exposure force, duration, directionality, and frequency.

The working groups identified several outstanding research questions that need to be addressed by future clinical studies to understand and characterize better the development of CTE: (1) study design should consider and accommodate the latency of neurodegenerative pathological cascades and progression of clinical symptoms after blast exposures; (2) research needs to assess the influence of comorbidities (e.g., sleep disturbances, mental health conditions [e.g., PTSD], diabetes mellitus, metabolic conditions, substance abuse) on CTE progression; (3) designing future longitudinal studies according to common frameworks (e.g., sample size requirements, outcome measures, biomarkers) and common data elements would enable data to be compared between studies; and (4) existing research limitations need to be addressed, including recollection bias in current datasets and regulatory burdens (e.g., multiple, at times conflicting, institutional review boards [IRBs]).

The working groups noted that retrospective studies inherently convey limited information about exposure variables (e.g., severity, frequency), the natural history of symptoms, and comorbidities potentially contributing to neuropathology and/or symptoms. Future retrospective studies should use all available military medical records, although it was acknowledged that they are limited by a general lack of TBI data.

Future prospective studies should collect imaging data, blood, and cerebrospinal fluid (CSF) biomarker samples; development of biomarkers capable of identifying subconcussive injuries is particularly needed. For CSF biomarkers, methods for detecting nonsteroidal anti-inflammatory drugs and serotonin-selective reuptake inhibitors in patients would be valuable. Investigators also need to consider the limitations of lumbar CSF sampling and recognize differences in sampling ventricular CSF. Developing neuroimaging biomarkers also requires improved positron emission tomography (PET) and better quantitative analysis methods. Prospective studies should also incorporate sensors to measure the physical properties of blast exposure; the existing limitations of sensor technology create a need for the development of new sensorbased approaches. 
Question \#4: What approaches can be used to detect early stages of blast-related neurodegeneration and evaluate the progression to CTE to support the screening, detection, diagnosis, prognosis, assessment of therapeutic interventions, and determination of return to duty (RTD) status?

Is there a specific structure-function profile that can be established using a combination of imaging (diffusion tensor imaging [DTI] and/or PET) and clinical measures that would definitively identify CTE pre-mortem? The working groups noted that detection of blast-associated CTE development is not possible given existing knowledge. Neuropathological abnormalities and clinical presentation are poorly defined at early stages of CTE. In addition, differentiating between CTE and other neurodegenerative disorders (e.g., Alzheimer disease [AD]) is difficult. Given these realities, the validation of diagnostics is implausible. The working groups identified a number of potential approaches to support screening, detection, diagnosis, prognosis, and treatment of those with early-stage CTE (e.g., imaging, registries, electrophysiology, neuropsychological testing, genomic assessment) that need standardization and validation for stable use over longer-term research time frames and clinical applications. Further, there is no scientific evidence that existing DoD RTD guidelines, which are based on clinical symptoms, are protective against the risk of neurodegeneration.

The working groups agreed that development of pre-morbid biomarkers is needed for detection of blast-related neurodegeneration and early-stage CTE. Potential neuroimaging biomarkers would be enabled further by standardization of imaging acquisition protocols, establishment of normative datasets, and the development of innovative analytic approaches. Working groups indicated that improvement of PET ligands to image amyloid, tau, inflammation, and related pathways are needed. While effective and tolerable CSF biomarkers exist for AD and severe TBI, a widespread search for CSF biomarkers of CTE (including microribonucleic acid) is needed. Current blood plasma biomarker techniques do not distinguish tau from other nonbrain sources of the protein (e.g., muscle), so methods for detection of proteins derived from the central nervous system (e.g., exosomes) are needed.

The working groups agreed that determining structure-function relationships specific to early-stage CTE using imaging and clinical measures is not possible at this time given existing gaps. Current neuroimaging modalities do not distinguish CTE from AD or TBI reliably. Ongoing efforts to develop additional PET ligands, improve analytic techniques, and establish new physiological targets (e.g., neuroinflammation, vascular damage) potentially could enable identification of CTE with neuroimaging. Neurodegeneration in the cerebellum may be particularly relevant to CTE, so a focus on neuroimaging in this region in addition to assessment of cerebellarmediated functional outcomes was suggested.

\section{Question \#5: What are the strategies that can be used to prevent, mitigate, or treat neurodegeneration following repeated blast exposure?}

The working groups agreed that a better understanding of the progression of neurodegeneration after repeated blast exposure is crucial to developing prevention, mitigation, and treatment strategies. Continued study of blast-induced neurophysiological injury cascades leading to neurodegeneration, including neuroinflammatory processes, apoptosis, gliosis, autoimmune effects, and protein aggregation (tau, amyloid beta), will inform identification of targets for these strategies. Research should include developing animal models with standardized, scaled blast exposures and longterm follow-up.

The working groups also agreed that among existing efforts to develop strategies that would prevent blast-related injury, the most promising include improving personal protective equipment (PPE) and vehicle design, providing additional training, and establishing limits (number, frequency) for blast exposure. Several areas of research are needed to identify factors predicting neurodegeneration and development that can be targeted by prevention development efforts.

An improved understanding of blast physics and how physical forces measured by sensors relate to brain injury is needed. This understanding can be accomplished by enhancing field measurements of environmental exposure and developing additional computational and empirical tools for estimating biomechanical injury risk. Better characterization of biological dosimetry measures and improved surveillance and reporting of exposures during training and in the field (including the use of sensors) will inform development of new and existing protocols that limit blast exposure. Materials science approaches should be pursued to develop new and advanced solutions for PPE and vehicle design. Researchers should also examine the potential for altering training procedures and engagement tactics to maximize the ability of PPE to protect service members from blast exposure.

Although more work is needed to understand how injuries might be mitigated post-exposure and before the onset of neurodegeneration, several mitigation strategies were suggested. Working groups indicated that communication efforts within the military line that promote maximization of recovery time during the acute stage of injury (as much as possible given mission requirements) are an effective mitigation strategy. Communication strategies are needed to change attitudes about reporting TBI, to improve reporting of TBI, and to enable line leaders to comply with guidelines for reporting potentially compensable events (PCEs). Steps to maximize recovery at the acute stage of injury may include: (1) encouraging the longest period of rest after that is possible, given mission requirements; (2) assisting service members with lifestyle management; (3) researching the influence of exercise, diet, drug and alcohol use, and cognitive exercises on brain recovery after blast injury; (4) improving sleep/sleep hygiene, particularly during the early recovery period; (5) developing standards for progressive RTD; and (6) researching biological markers that might identify the onset and end of the risk period.

The working groups noted that more research is needed before treatments for those with blast-related neurodegeneration can be offered. Recommended lines of research include pharmacological targeting of specific molecular pathologies (e.g., tau, inflammation, apoptosis, glutamate excitotoxicity, amyloid beta, astrogliosis, microgliosis), improving data sharing access across agencies and resolving conflicts that arise as a result of having multiple IRBs, investigating the benefits of cognitive behavioral therapy tailored to specific symptom constellations, and promoting the benefit of a healthy lifestyle to maximize improvements in brain health after injury.

\section{Expert Panel Findings}

The expert panel noted that the majority of blast-related TBIs within the military population arise from a mixed exposure to both blast overpressure and impact as opposed to blast overpressure alone; this complicates the meaning of "blast" when discussing blast-related trauma. The pathology of TBIs and any subsequent neurodegenerative outcomes arising from either of these exposure types is largely unknown. As a result, the overarching finding identified by the expert panel is that existing scientific evidence is insufficient to link blast-related TBI with CTE. The expert panel 
identified 13 findings describing research and knowledge gaps, clinical gaps, and research opportunities that, if addressed with focused effort, would further the understanding of the potential relationship between blast-related trauma and CTE.

\section{Research and knowledge gaps}

1. There is a lack of blast-exposed clinical tissue, with wellannotated medical and blast exposure histories, available for neuropathological analysis. Limited opportunity to gather neuropathological evidence with historical data impedes the ability to explore the association between neuropathology and lifetime risk factors or clinical features. Better understanding of the human neuropathology of blast and any associated neurodegeneration is needed to develop informative animal models.

2. Standard definitions of "blast" and "blast exposure" are needed. Blast-related exposures can encompass all types of blast injury, from primary to quinary. Different pre-clinical and clinical investigations model or define blast differently, however, which introduces variability across studies and limits reproduction and validation of findings.

3. Existing animal models are inadequate to study blast-related TBI and neurodegeneration. Validated and clinically relevant animal models are needed to identify underlying mechanisms of acute and chronic blast injury. Absent an understanding of injury mechanisms, the discovery of diagnostics and treatments is greatly inhibited.

4. Longitudinal and prospective studies, both having a strong neuropathological component, are needed to characterize the risk factors and spatiotemporal development of CTE. Informed by the current state-of-the-science, well-designed studies that collect detailed medical histories and exposures (frequency, magnitude) from service members and veterans and obtain consent for brain donation by these individuals can help establish the role of blast-related trauma in the development of CTE.

5. Limitations to data access and data sharing impose significant barriers to research. Legal, ethical, and logistical barriers to use of service member and civilian health data, exposure history, and medical records prevent analysis of potentially useful clinical information. Promoting broader access and use of these extant data could result in rapid answers to outstanding questions on the potential relationship between blast exposure and chronic TBI.

6. Substantiated risk factors for CTE are unknown, with the exception of exposure to head trauma. Studies of other potential risk factors, including gender, age, psychological health, drug or alcohol abuse, and genetics, have not been conclusive.

7. Evidence-based RTD protocols are lacking. There is no existing scientific evidence establishing parameters of existing DoD RTD guidelines, which are based on clinical symptoms, as protective against the risk of neurodegeneration.

\section{Clinical gaps}

8. Clear and standardized clinical criteria for CTE are lacking, impeding progress in development of pre-mortem diagnosis and screening approaches. Because the clinical features associated with CTE overlap with other neurodegenerative conditions such as AD, identification of specific or unique clinical diagnostic criteria has been difficult. Standardized neuropsychological and behavioral assessments relevant to
CTE are also lacking. Definition and diagnosis of CTE should be driven ultimately by clinicopathological approaches that incorporate clinical and pathological information. Defining and describing CTE as a spectrum disease, rather than as a binary condition, should also be considered.

9. Development of fluid and imaging biomarkers are needed for diagnosis of CTE and treatment of those with CTE. Noninvasive biomarkers of CTE would enable pre-mortem diagnosis and serve to assess the efficacy of therapeutic strategies.

10. Current neuroimaging approaches cannot diagnose CTE or distinguish CTE from other neurodegenerative disorders. Neuropathological abnormalities associated with CTE, such as tau or $\mathrm{A} \beta$ protein aggregation, cannot be characterized reliably with existing neuroimaging technology. Spatial resolution of existing neuroimaging modalities is also insufficient to image neuropathology at the scale observed in CTE studies.

\section{Research opportunities}

11. Extant data can be used to investigate CTE risk factors. Clinical correlation studies of electronic health record data and/or concussion care clinics should be explored.

12. Populations exposed to blast should be monitored continuously. Monitoring may help to determine a dose-response relationship between blast intensity and frequency and severity of human injury. Once a dose-response curve is established in humans, it will become easier to create more accurate animal models of blast injury.

13. Sensor technology development and materials science offer significant research and prevention opportunities. The PPEembedded blast wave sensors could allow objective measurement to supplement, or replace, exposure documentation. Sensor-enabled detection of rotational acceleration, which may play a role in blast-related mid-brain and cerebellar injury, may be valuable. Advanced materials research can benefit sensor technology development, as well as personal and vehicular protection.

\section{Expert Panel Recommendations}

An additional goal of the 2015 SoS meeting was to develop recommendations to advance the state of the science on the potential relationship between blast-related trauma and CTE. The expert panel identified six recommendations describing specific actions needed to advance research exploring the potential relationship between blast-related trauma and CTE. The six recommendations, with the first four characterized in order of highest priority for addressing pressing research needs, include:

- First, creation of a coordinated brain bank and tissue repository system is recommended with a robust plan for donation of clinical specimens that are annotated with medical and blast exposure data; this would enable exploration of the relationship between neuropathology and risk factors or clinical features.

- Second, development of standardized clinical diagnostic criteria for CTE is recommended to enable pre-mortem identification of the condition, ideally in concert with neuropathological information.

- Third, development and validation of clinically relevant animal models is recommended to explore potential biological mechanisms linking blast injury and development of CTE. 
1. Create a coordinated brain bank/repository system, accessible to the research community.

2. Develop standardized clinical diagnostic criteria.

3. Develop and validate animal models clinically relevant to blast injury and chronic neurodegeneration.

4. Develop a strategy for development of nextgeneration biospecimen and imaging biomarkers.

5. Strengthen ongoing longitudinal and prospective studies, or initiate new studies, to explore the spatiotemporal development of CTE and candidate risk factors, respectively; emphasize neuropathological analysis of blast-exposed tissue.

6. Implement prevention and mitigation strategies until treatment strategies become available
Short term (1-2 years)

Long term (5+ years)

Immediate $(<1$ year $)$

Short term (1-2 years)

Mid-term (3-4 years)

Long term (5+ years)

(1-2 years)

Short term (1-2 years)

Immediate $(<1$ year $)$

Short term (1-2 years) Mid-term (3-4 years)

Long term (5+ years)

Immediate $(<1$ year $)$

Short term (1-2 years)

Long term $(5+$ years $)$

Immediate $(<1$ year $)$

Long term (5+ years)
- Educate researchers on the importance of neuropathology and provide communication strategies that would enable them to advocate for brain donation with participants and families.

- Address logistical barriers of brain donation to promote rapid acquisition of brain tissue and facilitate sharing within research community.

- Establish a central repository and laboratory for storing, processing, and analyzing brains.

- Collect 100 brains

- Develop definitive criteria for establishing the pathology of blast exposure.

- Collect 1000 brains, including medical and blast exposure history.

$\circ$ For brains acquired $<48 \mathrm{~h}$ post-mortem, fix one hemisphere (e.g., for immunostaining) and freeze the other hemisphere (e.g., for in situ hybridization).

- For brains acquired $>48 \mathrm{~h}$ post-mortem, fix both hemispheres.

- Gather exposure data from pre-enlistment and pre- and postdeployment time periods.

- Use standardized neuropsychological/behavioral assessments validated for serial use in subjects with suspected blast-related neurodegeneration.

- Continue development of biospecimen biomarkers of injury to complement exposure histories.

- Follow subjects with blast exposure, with potential exposure, and agematched controls long term to determine the incidence of blastinduced neurodegeneration.

- Develop standardized protocols with blast exposure characteristics.

- Measure outcomes at longer time points after blast exposure that more appropriately reflect chronic effects.

- Share protocols across the research community.

- Convene an expert panel to assess current biomarkers and make recommendations for future development of technologies, protocols, and analytics.

- Develop a long-term plan for standardizing biomarker measurement.

- Explore combinatorial imaging modalities for differential diagnosis.

- Develop high spatial resolution neuroimaging approaches to detect potential neuroanatomical characteristics specific to CTE.

- Convene an objective expert panel to:

- Identify gaps in current longitudinal studies that can be addressed with additional data (e.g., neuropathological tissue).

- Review current prospective studies to determine wheher risk factors are addressed adequately.

- Plan a prospective study using candidate risk factors, potential biomarkers, and tissues that would address remaining gaps.

- Identify additional potential risk factors for analysis that may include genetic susceptibility, gender, age, drug abuse, and performanceenhancing drugs

- Initiate recruitment and data collection, with an emphasis on neuropathological analysis of blast-exposed tissue.

- Analyze prospective data to determine which candidate risk factors and biomarkers can be quantified.

- Modify training protocols to reduce exposure (without reducing training time).

- Educate military instructors about long-term exposure risks.

- Maintain optimal brain health in service members (e.g., sleep, etc.).

- Reinforce existing guidelines for management of mTBI.

- Improve equipment (PPE and vehicles) to mitigate injury.

- Determine extent to which RTD guidelines mitigate risk of neurodegeneration.

PPE, personal protectve equipment; RTD, return to duty. 
- Fourth, development of biomarkers is recommended to enable pre-mortem diagnosis and study of CTE in living persons.

- Fifth, increasing implementation of ongoing longitudinal studies and initiation of new prospective studies to assess candidate risk factors of CTE as well as spatiotemporal development of CTE and other candidate neuropathological changes linked to long-term sequelae resulting from blast exposure.

- Sixth, increasing implementation of existing prevention and mitigation strategies in at-risk or exposed populations, given that treatment strategies for CTE are not yet available.

For additional details on specific immediate ( $<1$ year), short term (1-2 years), mid-term (3-4 years), and long-term (5+ years) timeframes for addressing necessary components of these recommendations, see Table 1 .

\section{Conclusion}

The 2015 SoS meeting sought to comprehensively survey existing evidence and gaps in knowledge concerning the potential relationship between blast-related trauma and CTE. The Expert Panel identified one overarching finding, followed by 13 findings, including research and knowledge gaps, clinical gaps, and potential research opportunities. The overarching finding is that existing scientific evidence is insufficient to link blast-related TBI with CTE.

Seven of the subsequent expert panel findings described research and knowledge gaps, which include a lack of (1) standard definitions of blast and blast exposure; (2) adequate access to blastexposed clinical tissue with well-annotated medical and exposure information; (3) validated and clinically relevant animal models; (4) longitudinal and prospective studies with neuropathological components to characterize the risk factors and spatiotemporal development of CTE; (5) data access and data sharing within the scientific community; (6) substantiated risk factors for CTE, other than head trauma; and (7) evidence-based RTD guidelines.

Three expert panel findings identified clinical gaps that include a lack of (1) clear and standardized clinical diagnostic criteria for CTE; (2) fluid and imaging biomarkers for diagnosis of CTE and treatment of those with CTE; and (3) neuroimaging approaches capable of diagnosing CTE or distinguishing CTE from other neurodegenerative disorders. Finally, three expert panel findings identified potential research opportunities, including (1) use of extant data to investigate CTE risk factors; (2) continuous monitoring of populations exposed to blast, to explore dose-response relationships between blast intensity and injury severity; and (3) sensor technology development and materials science to advance data gathering and prevention strategies.

An additional goal of the 2015 SoS meeting was to develop recommendations to advance the state of the science on the potential relationship between blast-related trauma and CTE. The expert panel identified six recommendations, the first four of which were determined as highest priority for addressing pressing research needs. The first four recommendation in order of priority include (1) more collection and study of clinical neuropathology samples, (2) standardization of clinical diagnostic criteria, (3) development of clinically appropriate and standardized animal models, and (4) development of noninvasive serial assessment strategies (i.e., imaging or biospecimen biomarkers). Findings and recommendations identified by the 2015 SoS meeting can help develop methods and tools for achieving a better understanding of the potential relationship between blast-related trauma and CTE.
This knowledge could translate into enhanced strategies for prevention, mitigation, diagnosis of CTE and treatment of those with CTE or other blast-induced neurodegeneration experienced by service members.

\section{Author Disclosure Statement}

No competing financial interests exist.

The views expressed in this article are those of the authors and do not necessarily reflect the official policy or position of the Department of the Department of Defense, nor the U.S. Government. This work was prepared as part of the official duties of employee of the U.S. Government including military members. Title 17 U.S.C. $\S 105$ provides that 'Copyright protection under this title is not available for any work of the United States Government.' Title 17 U.S.C. $§ 101$ defines a U.S. Government work as a work prepared by a military service member or employee of the U.S. Government as part of that person's official duties.

\section{References}

1. Hazrati, L.N., Tartaglia, M.C., Diamandis, P., Davis, K.D., Green, R.E., Wennberg, R., Wong, J.C., Ezerins, L., and Tator, C.H. (2013). Absence of chronic traumatic encephalopathy in retired football players with multiple concussions and neurological symptomatology. Front. Hum. Neurosci. 7, 222.

2. Karantzoulis, S. and Randolph, C. (2013). Modern chronic traumatic encephalopathy in retired athletes: what is the evidence? Neuropsychol. Rev. 23, 350-360.

3. McCrory, P., Meeuwisse, W.H., Kutcher, J.S., Jordan, B.D., and Gardner, A. (2013). What is the evidence for chronic concussionrelated changes in retired athletes: behavioural, pathological and clinical outcomes? Br. J. Sports Med. 47, 327-330.

4. Randolph, C. (2014). Is chronic traumatic encephalopathy a real disease? Curr. Sports Med. Rep. 13, 33-37.

5. Wortzel, H.S., Brenner, L.A., and Arciniegas, D.B. (2013). Traumatic brain injury and chronic traumatic encephalopathy: a forensic neuropsychiatric perspective. Behav. Sci. Law 31, 721-738.

6. Hinds, S. (2014). Subject: Chronic Traumatic Encephalopathy. Def. Veterans Brain Inj. Cent. https://dvbic.dcoe.mil/tbi-research-reviews (last accessed July 17, 2015).

7. McKee, A.C., Stern, R.A., Nowinski, C.J., Stein, T.D., Alvarez, V.E., Daneshvar, D.H., Lee, H.S., Wojtowicz, S.M., Hall, G., Baugh, C.M., Riley, D.O., Kubilus, C.A., Cormier, K.A., Jacobs, M.A., Martin, B.R., Abraham, C.R., Ikezu, T., Reichard, R.R., Wolozin, B.L., Budson, A.E., Goldstein, L.E., Kowall, N.W., and Cantu, R.C. (2013). The spectrum of disease in chronic traumatic encephalopathy. Brain 136, 43-64.

8. McKee, A.C., Stein, T.D., Kiernan, P.T., and Alvarez, V.E. (2015). The neuropathology of chronic traumatic encephalopathy. Brain Pathol. 25, 350-364.

9. Omalu, B., Bailes, J., Hamilton, R.L., Kamboh, M.I., Hammers, J., Case, M., and Fitzsimmons, R. (2011). Emerging histomorphologic phenotypes of chronic traumatic encephalopathy in American athletes. Neurosurgery 69, 173-183.

10. Riley, D.O., Robbins, C.A., Cantu, R.C., and Stern, R.A. (2015). Chronic traumatic encephalopathy: Contributions from the Boston University Center for the Study of Traumatic Encephalopathy. Brain Inj. 29, 154-163.

11. Saigal, R. and Berger, M.S. (2014). The long-term effects of repetitive mild head injuries in sports. Neurosurgery 75, Suppl 4, S149-S155.

Address correspondence to: Raj K. Gupta, PhD

Department of Defense Blast Injury Research Program Coordinating Office

United States Army Medical Research and Materiel Command 504 Scott Street

Fort Detrick, MD 21702

E-mail: raj.k.gupta.civ@mail.mil 